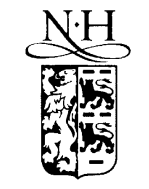

ELSEVIER

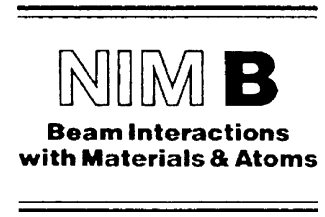

www.elsevier.com/locate/nimb

\title{
Profiles and parameters of tracks in the LR115 detector irradiated with alpha particles
}

\author{
D. Nikezic ${ }^{1}$, K.N. Yu ${ }^{*}$ \\ Department of Physics and Materials Science, City University of Hong Kong, Tat Chee Avenue, \\ Kowloon Tong, Kowloon, Hong Kong, China
}

Received 26 February 2002; received in revised form 22 May 2002

\begin{abstract}
The three-dimensional and analytical theory for track growth in solid state nuclear track detector is incorporated in a computer program for calculating track parameters and for plotting track-wall profiles and contours of the track opening in the LR115 detector irradiated by alpha particles. Some differences between the behavior of CR39 and LR115 detectors are discussed. Track parameters as well as the major and minor axes of the track openings have been calculated as a function of the energy and incident angle of the alpha particles. Vertical track profiles are given for incident angles between $20^{\circ}$ and $90^{\circ}$ and energies from 1 to $4 \mathrm{MeV}$, while the contours of track openings are given for incident angles from $10^{\circ}$ to $90^{\circ}$ and energies from 1 to $5 \mathrm{MeV}$. The track profiles and contours shown in the present study represent the tracks without any distortion.
\end{abstract}

(c) 2002 Published by Elsevier Science B.V.

Keywords: LR115 detector; Major and minor axes; Track profile; Track opening

\section{Introduction}

The growth of tracks in solid state nuclear track detectors (SSNTDs) have been studied by many investigators [1-7]. During etching, the track passes through different phases which have been fully described and characterized in [8]. The common characteristic of the investigations mentioned

\footnotetext{
${ }^{*}$ Corresponding author. Tel.: +852-2788-7831; fax: +8522788-7830.

${ }^{1}$ On leave from Faculty of Sciences, University of Kragujevac, 34000 Kragujevac, Yugoslavia.
}

above is that the tracks have been considered as two-dimensional objects. The only exception is [8] in which some treatments of the tracks as threedimensional objects were given, but only the minor axes of the track openings were calculated.

A three-dimensional and analytical model for track development in SSNTDs was developed recently $[9,10]$. The equations derived in the model enable calculations of track parameters, as well as the plotting of track profiles and contours of track openings. The track parameters, profiles and contours have been calculated and plotted for the CR39 detector irradiated with alpha particles [11]. Another frequently used SSNTD is the LR115 
detector. Descriptions of these two detectors and their usage in radon measurements and related fields have been summarized [12].

In the present paper, we present calculations of track parameters, plots of contours of track openings and wall profiles for the LR115 detector irradiated by alpha particles. There are some important differences between the CR39 and LR115 detectors. The first important difference lies in the thickness of the active layer, which is $12 \mu \mathrm{m}$ for the LR115 type II detector and about $0.5 \mathrm{~mm}$ for the CR39 detector. The LR115 detector is such a thin film that the alpha-particle tracks appear in the form of perforated holes, which is not the case for the CR39 detector.

Furthermore, the LR115 detector is based on cellulose nitrate while the CR39 detector is based on polycarbonate materials. Due to the difference in the base materials, there are also differences in the variations of the track etch rate $V_{\mathrm{t}}$ with the residual range of alpha particles in the detector material, $R^{\prime}$, which are shown in Fig. 1 (note the logarithmic scale on the ordinate axis). The curves in Fig. 1 were generated according to the following formulae [13]:

$$
\begin{aligned}
V= & 1+\left(11.45 \mathrm{e}^{-0.339 R^{\prime}}+4 \mathrm{e}^{-0.044 R^{\prime}}\right) \\
& \times\left(1-\mathrm{e}^{-0.58 R^{\prime}}\right), \quad \text { for CR39 detector, } \\
V= & 1+\left(100 \mathrm{e}^{-0.446 R^{\prime}}+5 \mathrm{e}^{-0.107 R^{\prime}}\right) \\
& \times\left(1-\mathrm{e}^{-R^{\prime}}\right), \quad \text { for LR115 detector, }
\end{aligned}
$$

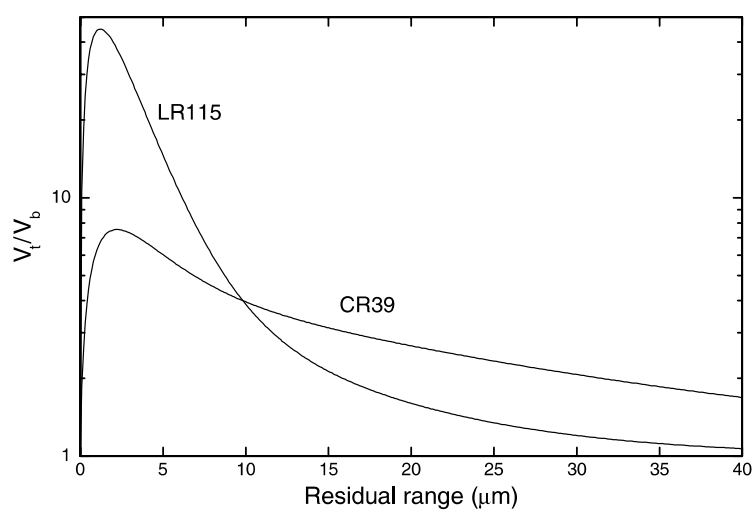

Fig. 1. $V_{\mathrm{t}} / V_{\mathrm{b}}$ as a function of the residual alpha particle energy in the CR39 and the LR115 detectors. where $V=V_{\mathrm{t}} / V_{\mathrm{b}}$ is the ratio of the track etch rate $V_{\mathrm{t}}$ to the bulk etch rate $V_{\mathrm{b}}$.

The graphs in Fig. 1 explain the difference in sensitivity to alpha particles between the CR39 and the LR115. When the incident energy of an alpha particle is large (corresponding to a large residual range), the ratio $V_{\mathrm{t}} / V_{\mathrm{b}}$ is very close to 1 for the LR115 detector, and track formation is not possible. For the CR39, however, the $V_{\mathrm{t}} / V_{\mathrm{b}}$ is well above 1 , so tracks can be formed. In other words, some alpha particles that strike the LR115 detector with larger incident energies will not be detected, which will inevitably diminish the sensitivity of this detector. However, in the region of smaller energies, the ratio $V_{\mathrm{t}} / V_{\mathrm{b}}$ is much larger for the LR115 than for the CR39, which means the etching solution will penetrate along the particle trajectory much faster in the LR115 than in the CR39. From this graph one can predict narrower tracks in LR115 than in CR39 for the same incident conditions. It is apparent from the discussion here that the track characteristics and parameters are different in the CR39 and the LR115 detectors.

\section{Main equations of the three-dimensional track growth model}

Here, only the main equations of the model are presented below, but only briefly and without derivations. Full descriptions and derivations are given in [10].

\subsection{Sharp phase of track development}

In this phase on track development, the etching solution does not reach the full particle range in the detector. The track tip is sharp (which explains the term "sharp phase") and the track wall is semiconical. The track will be a regular cone if the track-etch rate $V_{\mathrm{t}}$ is constant. The equation of the track wall in two dimensions was derived based on the "tangent method" [14] as

$y=\int_{z}^{L} \frac{\mathrm{d} R^{\prime}}{\sqrt{V^{2}\left(R^{\prime}\right)-1}}$,

where $(z, y)$ represent the coordinates of points on the track wall, the $z$ axis is along the particle tra- 
jectory and $L$ is the etching depth along the particle trajectory in the detector material.

The equation for the track wall in the sharp phase in three dimensions for normal incidence was given as

$\sqrt{x^{2}+y^{2}}=\int_{z}^{L} \frac{\mathrm{d} R^{\prime}}{\sqrt{V^{2}\left(R^{\prime}\right)-1}}$,

where the $z$ axis is again along the particle trajectory and $(x, y)$ represent the coordinates of points on the track wall [9]. The track opening is circular in shape for normal incidence, but some are egglike, droplet-like, or even more complicated in shape for oblique incidence. The equation for the contour line of the track opening for an oblique incidence was obtained as

$\sqrt{\xi^{2}+(\eta \sin \theta)^{2}}=\int_{\eta \cos \theta+h / \sin \theta}^{L} \frac{\mathrm{d} R^{\prime}}{\sqrt{V^{2}\left(R^{\prime}\right)-1}}$,

where $\theta$ is the incident angle with respect to the surface of the detector, $(\xi, \eta)$ are coordinates of points on the track opening contour in the plane which represents the surface of the detector after etching and $h$ is the thickness of the removed layer.

\subsection{Rounded phase of track development}

When the etching solution passes over the range of the alpha particle in the detector material, a spherical structure is formed around the ending point and the track has a rounded tip. This is called the rounded or over-etched phase of track development. The equation of the track wall in this phase for normal incidence in two dimensions is

$y(x)=\int_{z-d \sin \delta}^{R} \frac{\mathrm{d} R^{\prime}}{\sqrt{V^{2}\left(R^{\prime}\right)-1}}+d \cos \delta$,

where $d$ is the over-etched thickness, i.e. the layer removed after the etching solution passes through the alpha-particle range and $\delta=\operatorname{asin}\left(1 / V\left(R^{\prime}\right)\right)$ the local developing angle of the track [10]. The equation of the track wall (also for normal incidence) in three dimensions is given analogously to Eq. (4) as

$\sqrt{x^{2}+y^{2}}=\int_{z-d \sin \delta}^{R} \frac{\mathrm{d} R^{\prime}}{\sqrt{V^{2}\left(R^{\prime}\right)-1}}+d \cos \delta$.
The equation of the contour line of the opening for an oblique incident angle $\theta$ was derived as

$$
\begin{aligned}
\sqrt{\xi^{2}+\eta^{2} \sin ^{2} \theta}= & \int_{z_{0}+\eta \cos \theta-d \sin \delta}^{R} \frac{\mathrm{d} R^{\prime}}{\sqrt{V^{2}\left(R^{\prime}\right)-1}} \\
& +d \cos \delta,
\end{aligned}
$$

where $(\xi, \eta)$ are coordinates of points on the contour line of the track opening in the plane of the detector surface after etching.

\section{Calculation method}

The calculation method applied here is similar to the one used before for the CR39 detector. The programs written earlier for CR39 were adapted for the LR115 detector. This task was not trivial because the small thickness of the LR115 detector required some modifications of the model. Alpha particles with energies larger than $3 \mathrm{MeV}$ can pass through the detector material and exit on the opposite side of the sensitive layer, i.e. the ending point of the particle range lies outside the detector material. In this case, formation of the spherical part of the track (with the center at the hypothetical ending point of the particle trajectory) will not occur. Such tracks will conserve their semiconical wall shape regardless of the etching duration, which is of course limited by the small thickness of the detector. For oblique incident angles, the track openings will maintain the ellipselike shape and cannot transform into the circular form. However, for normal incident angles, the openings are always circular.

If the alpha particles have energies smaller than $3 \mathrm{MeV}$, they can be completely stopped in the detector, and formation of the spherical part of the tracks is possible. In this case, the contours of the track openings can be ellipse-like. However, they can also be more complicated curves joining an ellipse with a circle. If the etching is sufficiently long and the energy is small, the opening can transform completely into the circular shape.

Unlike the case for the LR115, alpha particles emitted by natural radionuclides are always stopped in the CR39 detector, and formation of the spherical part will always occur in the CR39 after prolonged etching. 
In addition, very large ratios of $V_{\mathrm{t}} / V_{\mathrm{b}}$ in the LR115 in the low energy region cause some numerical problems which were not encountered for the CR39. However, detailed descriptions of such particulars are out of the scope of the present study. All the dissimilarities between the CR39 and LR115 mentioned above have been taken into account in the course of modifying the programs.

Calculations were carried out for the standard etching conditions that are usually applied to the LR115 detector, namely, the etchant of the $2.5 \mathrm{~N}$ aqueous solution of $\mathrm{NaOH}$ kept at $60^{\circ} \mathrm{C}$ and an etching duration of $2 \mathrm{~h}$. Under these etching conditions, the removed layer is $(3.27 \pm 0.08) \mu \mathrm{m} / \mathrm{h}$ [15], so the removed layer for $2 \mathrm{~h}$ of etching is 6.54 $\mu \mathrm{m}$, and the remaining thickness of the sensitive layer is $12-6.54=5.46 \mu \mathrm{m}$.

\section{Results}

Fig. 2 presents the relationship between the major axis of the track opening and the incident alpha particle energy, with the incident angle (with respect to the detector surface) as a parameter. Here, all tracks have been taken into account, regardless of whether they have been completely etched through the detector sensitive layer. It is interesting to note that the lines for the incident

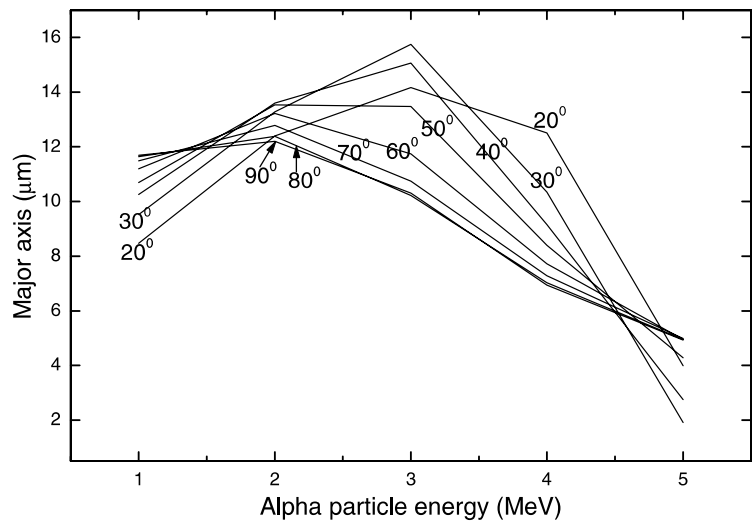

Fig. 2. Relationship between the major axis of the track opening and the alpha particle energy, with the incident angle as a parameter.

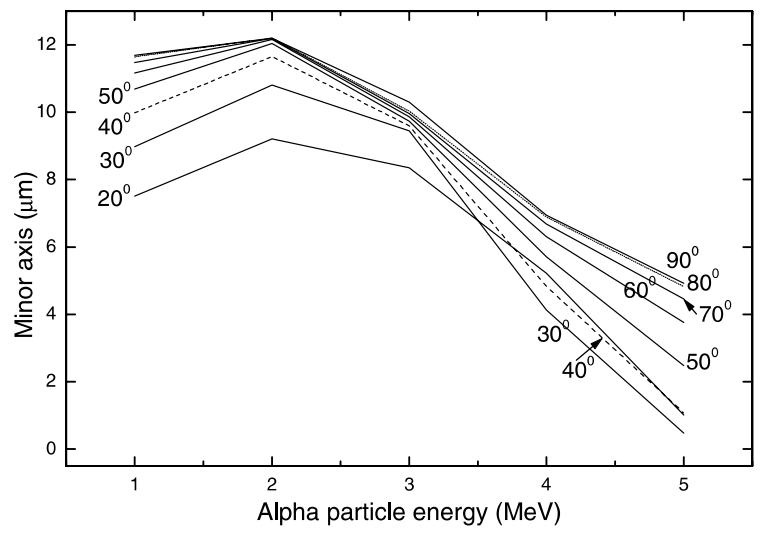

Fig. 3. Relationship between the minor axis of the track opening and the alpha particle energy, with the incident angle as a parameter.

angles between $70^{\circ}$ and $90^{\circ}$ are close to each other, i.e. the diameters of the tracks for larger incident angles depend only on the energy. In other words, the diameter of a circular track is a good measure of the incident energy. Fig. 3 gives the corresponding results for the minor axis of the track opening. Again, the curves for larger incident angles are close to each other. The minor axis also increases with the incident angle.

Figs. 4-7 represent the vertical track profiles for alpha energies of $1,2,3$ and $4 \mathrm{MeV}$ and for incident angles from $20^{\circ}$ to $90^{\circ}$. The images given here as well as those in Figs. 8-12 are directly plotted from FORTRAN90 files. The computer used for the calculations operated with the resolution of $640 \times 480$ pixels. In the program, the FORTRAN90 command STATUS $=$ SETWINDOW(.TRUE., -32,-24, 32, 24) is included to enable scaling of the computer screen to a dimension of $64 \times 48$. The first pair of numbers $(-32,-24)$ gives the coordinates of the lowest-left point, while the second pair $(32,24)$ gives the uppermost-right point of the screen. In this way the images obtained represent those obtained in the real world. The switch .TRUE. means that the ordinate is directed upwards (there are a number of possible coordinate systems in FORTRAN90 and users can choose the most convenient one for their uses).

Fig. 4 gives the track profile (or vertical crosssection of the track) in the LR115 detector when 


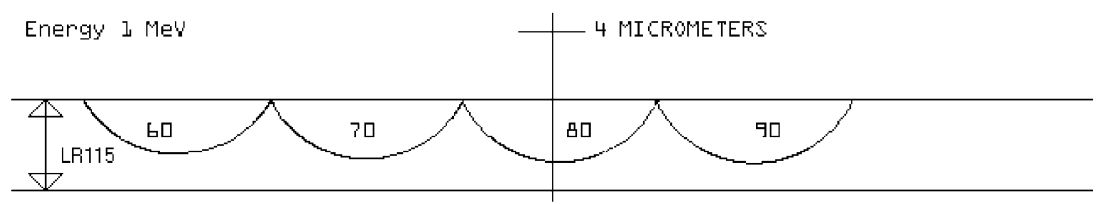

5.46 MICROMETERS

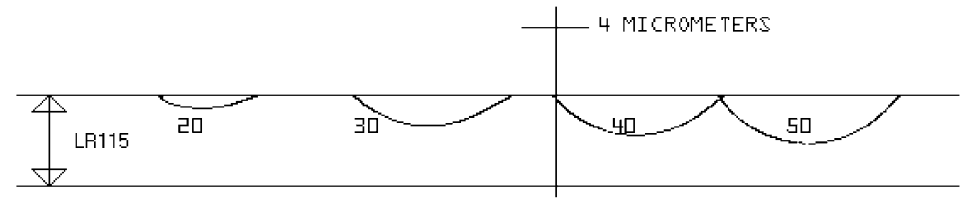

5. 46 MICROMETERS

Fig. 4. Track profiles for alpha particles with an incident energy of $1 \mathrm{MeV}$.

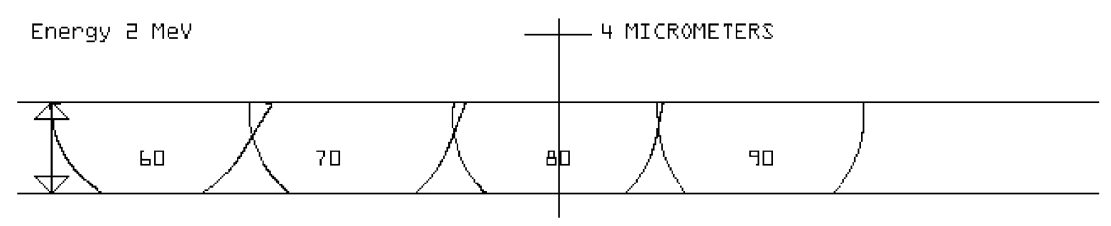

5. 46 MICROMETERS

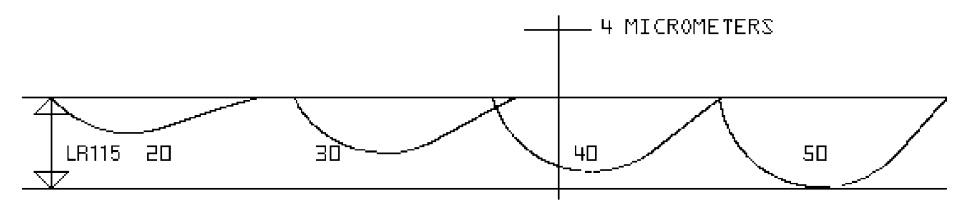

5.46 MICROMETERS

Fig. 5. Track profiles for alpha particles with an incident energy of $2 \mathrm{MeV}$.
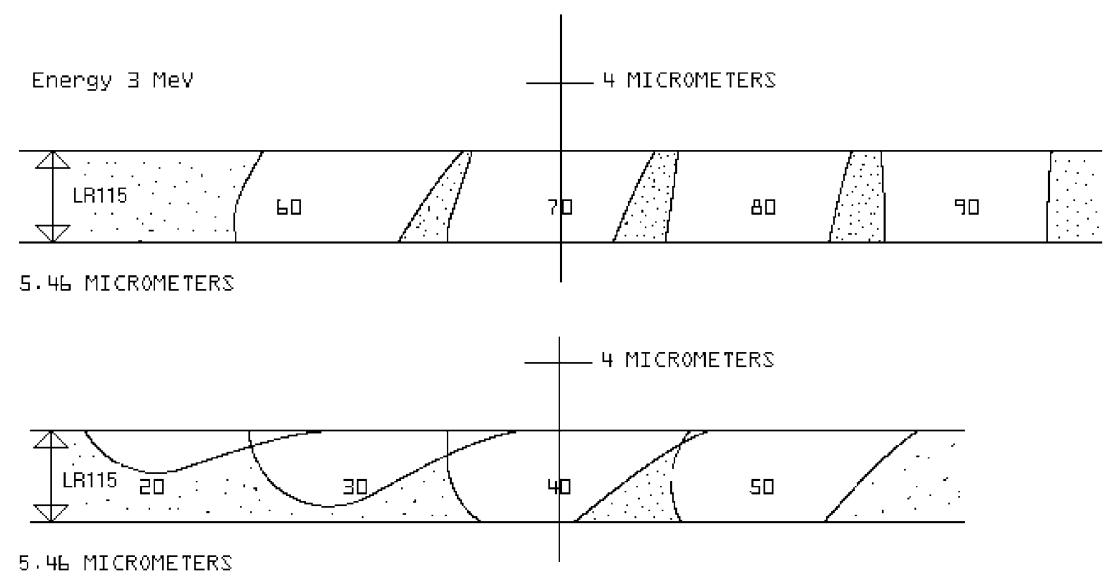

Fig. 6. Track profiles for alpha particles with an incident energy of $3 \mathrm{MeV}$. 

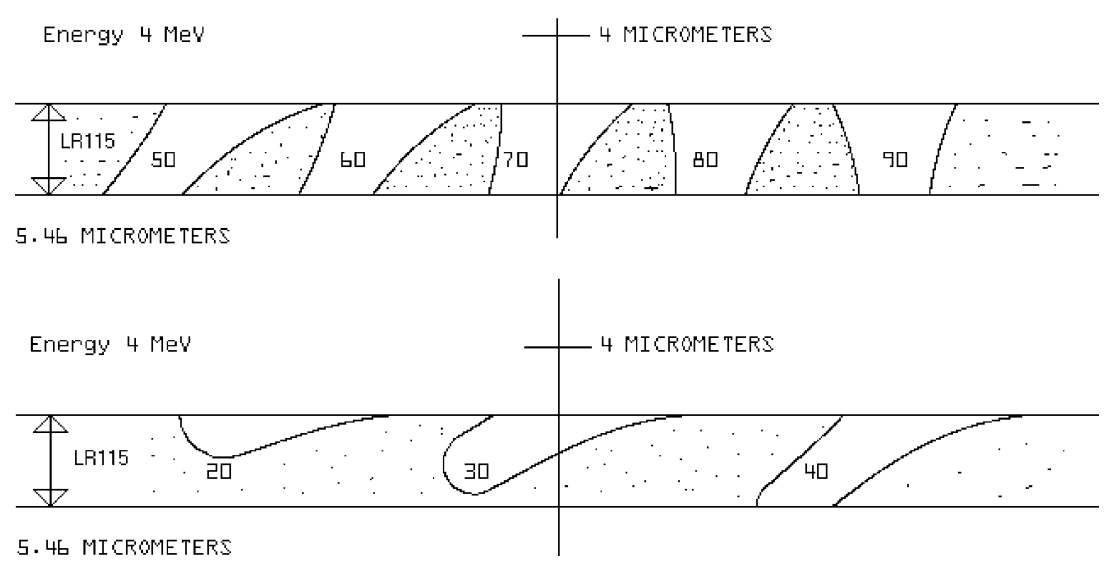

Fig. 7. Track profiles for alpha particles with an incident energy of $4 \mathrm{MeV}$.

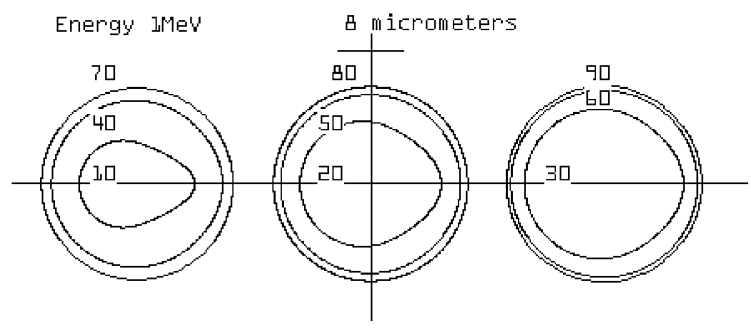

Fig. 8. Contours of track openings for alpha particles with an incident energy of $1 \mathrm{MeV}$.

the initial energy is $1 \mathrm{MeV}$. The upper horizontal line represents the detector surface after etching, and the lower horizontal line represents the bottom of the active layer which is adhered to the supporting plastic (not shown here). To enable scaling of the tracks, the mark " 4 micrometers" is shown on the figure (i.e. the distance from the upper horizontal line to the mark is $4 \mu \mathrm{m}$ ). The thickness $5.46 \mu \mathrm{m}$ is also shown in the figures, which represents the residual thickness of the LR 115 detector after etching. It can be observed in Fig. 4 that the tracks are mostly in a spherical shape (except those for incident angles of $20^{\circ}$ and $30^{\circ}$, which are slightly extended) and no tracks were etched completely through the sensitive layer. Alpha particles are stopped in the detector (i.e. they do not penetrate through it) and formation of the spherical part of the track is possible. If we "count" only those tracks for which the etchant penetrates through the detector, all the above particles would not be considered as detected. Under the optical microscope, however, light spots can be seen at the positions of these tracks.

Track profiles for an incident alpha energy of 2 $\mathrm{MeV}$ are given in Fig. 5. Tracks originated from particles with incident angles smaller than $50^{\circ}$ are

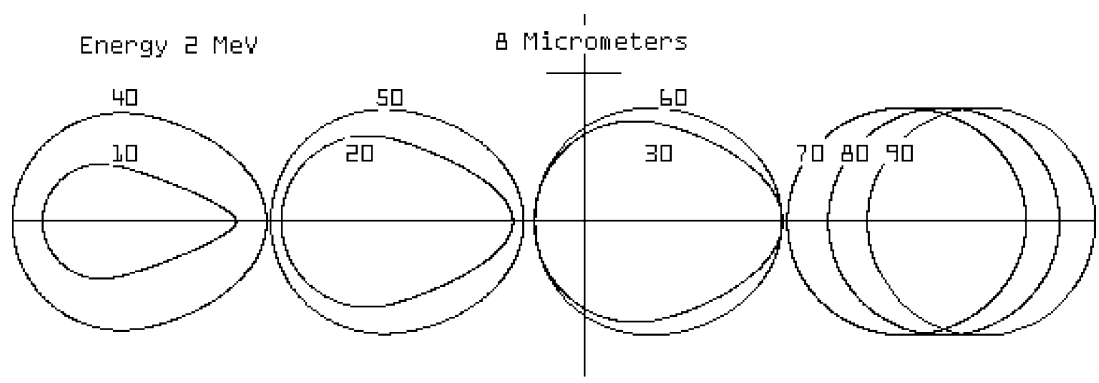

Fig. 9. Contours of track openings for alpha particles with an incident energy of $2 \mathrm{MeV}$. 


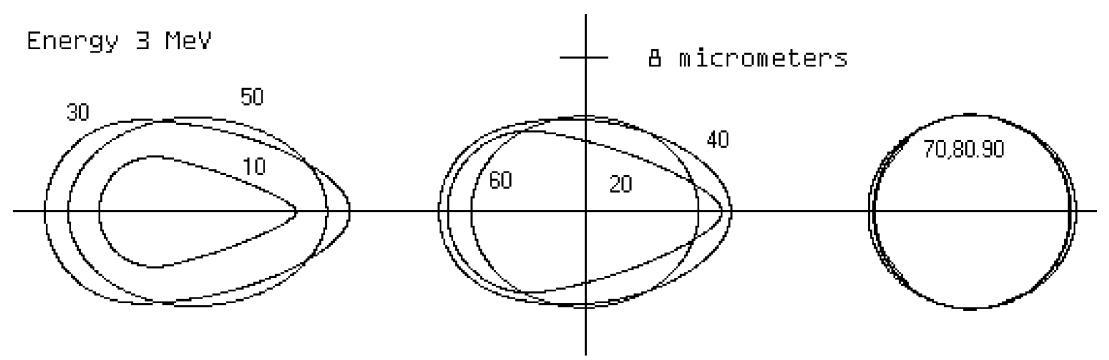

Fig. 10. Contours of track openings for alpha particles with an incident energy of $3 \mathrm{MeV}$.

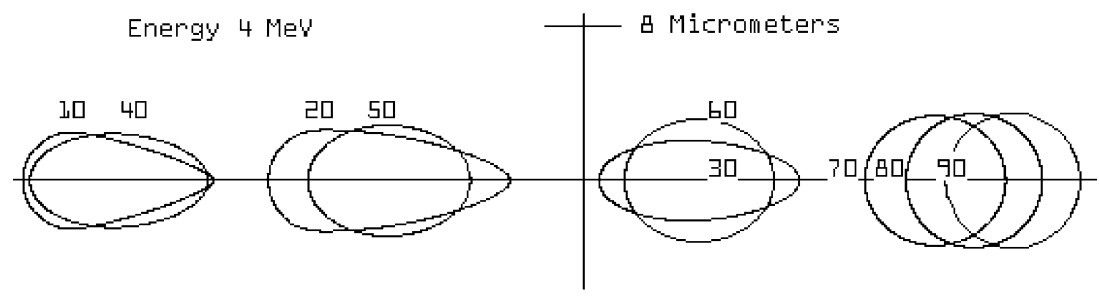

Fig. 11. Contours of track openings for alpha particles with an incident energy of $4 \mathrm{MeV}$.

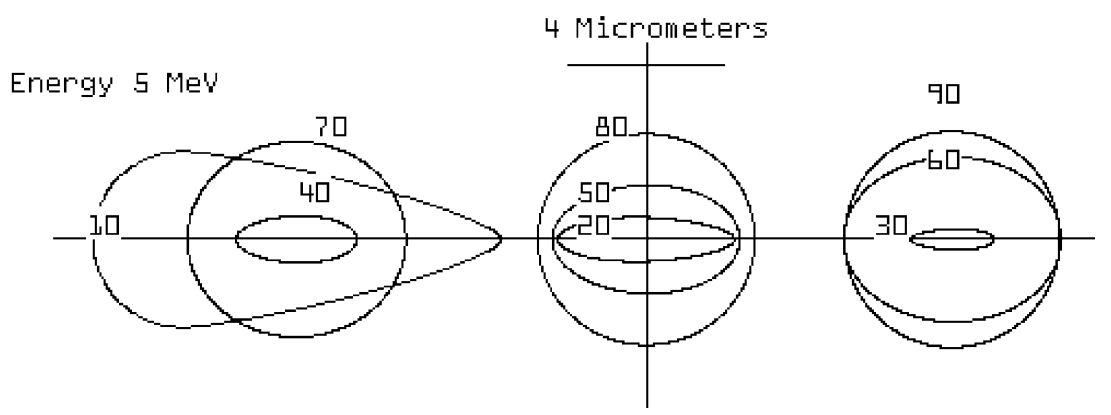

Fig. 12. Contours of track openings for alpha particles with an incident energy of $5 \mathrm{MeV}$.

not fully developed. Under the optical microscope light spots should be seen at their positions. For incident angles larger than $50^{\circ}$, the tracks are etched through the sensitive layer and bright light spots coming from the microscope lamp can be seen. Furthermore, these tracks are in spherical form, although the spheres are cut from both the top and the bottom (see Fig. 5).

Track profiles for incident alpha energies of 3 and $4 \mathrm{MeV}$ are shown in Figs. 6 and 7. The dotted areas represent the body of the detector. A variety of track profiles are presented in these figures.
Some tracks are overlapped with each other to save space only, and they can be used individually.

Figs. 8-12 give the contour of the track opening when the tracks are observed vertically from the above. The incident energy was varied from 1 to 5 $\mathrm{MeV}$ and the incident angle from $10^{\circ}$ to $90^{\circ}$ with increments of $10^{\circ}$. The incident angle is expressed as a parameter next to the corresponding contour line in the figures. The mark for $8 \mu \mathrm{m}$ is used in Figs. 8-11, and for $4 \mu \mathrm{m}$ in Fig. 12. Circular openings are typical for incident angles larger than $70^{\circ}$, and the track openings deviate more from 
being circular for smaller incident angles. All kinds of the track openings can be found in Figs. 8-12; circular for large incident angles, egg-like tracks and droplet-like tracks for very small incident angles. The general trend is that the diameter of the tracks decreases with the incident energy.

\section{Conclusions}

A previously developed three-dimensional and analytical theory for track growth was applied to the LR115 detector. The results presented here show that the theory is applicable for thin detectors like the LR115 with some modifications, which are mainly related to the position of the end point of the particle trajectory. If the end point lies outside of the detector material, the formation of the spherical part of the track is not possible. Other aspects of the theory are similar to these for a thick detector like the CR39, except for the different function of $V\left(R^{\prime}\right)$. The results given in this paper are similar to those presented in [16], where a slightly different method of track development was applied.

The track diameter of (essentially) the circular tracks depends mainly on the incident energy. This phenomenon makes the LR115 detector very convenient for the spectrometry of the alpha particles. With smaller incident angles, the tracks deviate more from being circular.

The accuracy of the calculations presented in this study depend on the accuracy of the functions $V\left(R^{\prime}\right)$ and $V_{\mathrm{b}}$.

\section{Acknowledgements}

The present research is supported by the CERG grant CityU1081/01P from the Research Grant Council of Hong Kong (City University of Hong Kong Ref. no. 9040639).

\section{References}

[1] P.R. Henke, E. Benton, Nucl. Instr. and Meth. 97 (1971) 483.

[2] G.H. Paretzke, E. Benton, P.R. Henke, Nucl. Instr. and Meth. 108 (1973) 73.

[3] G. Somogyi, Nucl. Instr. and Meth. 173 (1980) 21.

[4] M. Fromm, A. Chambaudet, F. Membrey, Nucl. Tracks and Radiat. Meas. 15 (1988) 115.

[5] U. Hatzialekou, D.L. Henshaw, A.P. Fews, Nucl. Instr. and Meth. A 263 (1988) 504.

[6] V. Ditlov, Radiat. Meas. 25 (1/4) (1995) 89.

[7] P. Meyer, M. Fromm, A. Chambaudet, J. Laugier, L. Makovicka, Radiat. Meas. 25 (1/4) (1995) 449.

[8] G. Somogyi, A.S. Szalay, Nucl. Instr. and Meth. 109 (1973) 211.

[9] D. Nikezic, Radiat. Meas. 32 (2000) 277.

[10] D. Nikezic, K.N. Yu, Radiat. Meas., submitted for publication.

[11] D. Nikezic, K.N. Yu, Radiat. Meas., submitted for publication.

[12] S.A. Durrani, R. Ilic, in: Radon Measurements by Etched Track Detectors: Applications in Radiation Protection, Earth Sciences and the Environment, World Scientific, Singapore, 1997.

[13] S.A Durrani, R.K. Bull, Solid State Nuclear Track Detection: Principles, Methods and Application, Pergamon Press, Oxford, 1987.

[14] D. Nikezic, D. Kostic, Radiat. Meas. 28 (1997) 185.

[15] D. Nikezic, A. Janicijevic, Appl. Radiat. Isot. 57 (2002) 275.

[16] R. Barillon, M. Fromm, A. Chambaudet, H. Marah, A. Sabir, Radiat. Meas. 28 (1997) 619. 